\title{
Methods for intraoperative, sterile pose-setting of patient-specific microstereotactic frames
}

Benjamin Vollmann, Samuel Müller, Dennis Kundrat, Tobias Ortmaier, Lüder A. Kahrs

Benjamin Vollmann, Samuel Müller, Dennis Kundrat, Tobias Ortmaier, Lüder A. Kahrs, "Methods for intraoperative, sterile pose-setting of patientspecific microstereotactic frames," Proc. SPIE 9415, Medical Imaging 2015: Image-Guided Procedures, Robotic Interventions, and Modeling, 94150M (18 March 2015); doi: 10.1117/12.2082066 


\title{
Methods for intraoperative, sterile pose-setting of patient-specific microstereotactic frames
}

\author{
Benjamin Vollmann, Samuel Müller, Dennis Kundrat, Tobias Ortmaier, Lüder A. Kahrs* \\ Institute of Mechatronic Systems, Leibniz Universität Hannover, \\ Appelstr. 11a, 30167 Hanover, Germany
}

\begin{abstract}
This work proposes new methods for a microstereotactic frame based on bone cement fixation. Microstereotactic frames are under investigation for minimal invasive temporal bone surgery, e.g. cochlear implantation, or for deep brain stimulation, where products are already on the market. The correct pose of the microstereotactic frame is either adjusted outside or inside the operating room and the frame is used for e.g. drill or electrode guidance. We present a patientspecific, disposable frame that allows intraoperative, sterile pose-setting. Key idea of our approach is bone cement between two plates that cures while the plates are positioned with a mechatronics system in the desired pose. This paper includes new designs of microstereotactic frames, a system for alignment and first measurements to analyze accuracy and applicable load.
\end{abstract}

Keywords: bone cement, targeted surgery, minimal-invasive cochlear surgery, deep brain stimulation, feasibility study

\section{INTRODUCTION}

Patient-specific instrumentation gains interest in various surgical disciplines. Different approaches for bone attached platforms for interventions around the head already exist and some of them are already commercialized or are under clinical testing ${ }^{1-3}$. Target objects are the cochlea, granuloma in the temporal bone, or anatomical structures inside the brain during interventions for deep brain stimulation. Nevertheless, transferring the individualization process (i.e. setting the patient-specific pose of such tools) into the operating room is still under investigation. Most approaches need a step of sterilization after fabrication or pose-setting, which is time-consuming, especially if a complete intraoperative workflow (including imaging) is targeted. Kratchman and Fitzpatrick invented a so-called Freeze Frame from which our methods branch off ${ }^{4}$. They suggested sterile 2-octyl cyanoacrylate to lock several mechanical sliding parts (joints) in a patient-specific position. During the experiments they used conventional superglue as substitute and performed the adjustment of the joints on a CNC milling machine. Additionally, they proposed a setup for intraoperative use to set the position of each leg consecutively with three degrees of freedom (DOF).

Our work describes further methods inside this research area and includes alternative concepts for patient-specific microstereotactic frames. The correct pose of the frame is set in a single step. Bone cement is filled in flexible, curvilinear legs without conventional joints. Additionally, a complete mechatronic setup for pose-setting is presented, which can be directly used inside the operating room (OR) close to the patient. Further advantages are e.g. less mechanical parts and easier mounting, smaller design, no milling, easier and smaller packaging, lightweight design and cost effectiveness.

\section{METHODS AND MATERIALS}

\subsection{Overall design}

Referred to the concept of a head-mounted device an universal frame comparable to the prepositioning frame of Kratchman et al. ${ }^{5}$ is created (see Figure 1a). An elastic strap, which is known from breathing masks and connected to the wings of the frame, surrounds the skull as shown in Figure 1b. Three sharpened spikes on the bottom surface prevent the frame from further movement and ensure stable contact to the skull. Three spherical fiducial markers are glued to the frame's top surface on defined positions that can either be measured by computed tomography (CT) or coordinate

\footnotetext{
* lueder.kahrs@imes.uni-hannover.de; phone +49 511762 4113; fax +49 511762 19976; www.imes.uni-hannover.de
}

Medical Imaging 2015: Image-Guided Procedures, Robotic Interventions, and Modeling,

edited by Ziv R. Yaniv, Robert J. Webster III, Proc. of SPIE Vol. 9415, 94150M

(C) 2015 SPIE · CCC code: 1605-7422/15/\$18 · doi: 10.1117/12.2082066 
measuring machines to generate reference points for a final trajectory. The patient-individual part (see Figure 1c) fits as counterpart in a defined orientation in the center of the universal frame and can be fixed by three sterilized screws. A burr shows the planned trajectory in Figure $1 \mathrm{~b}$.
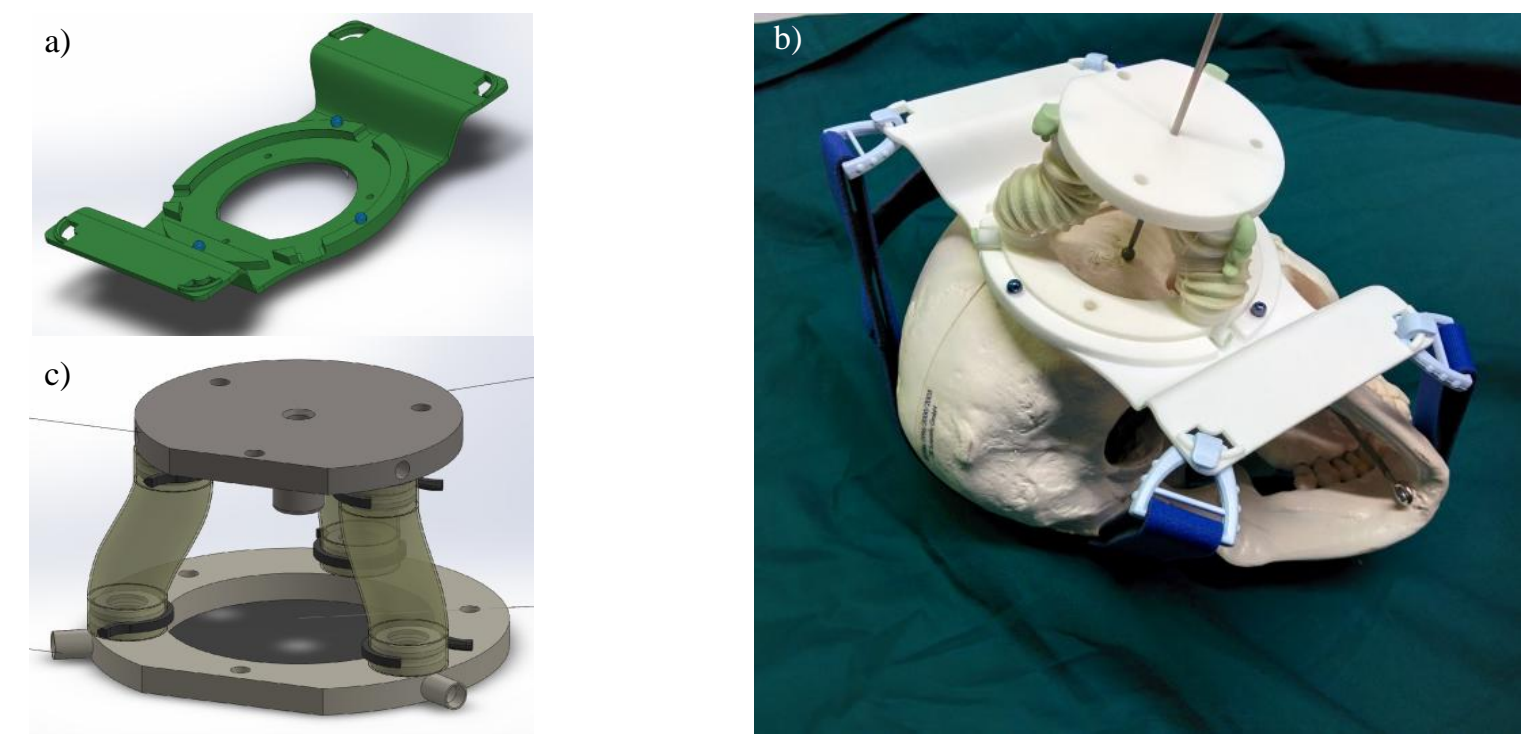

Figure 1. (a) Universal, head-mounted frame as CAD drawing. (b) Overall patient-individual microstereotactic frame mounted on a plastic skull with a Weinmann breathing mask strap. (c) Patient-individual part of the design.

\subsection{Components of patient-specific microstereotactic frame}

The design of our microstereotactic frames comprises disposable parts: Two independent plates connected by hoses with bone cement which harden in the operating room during the build process. The top and bottom plates are actually manufactured with rapid prototyping techniques and could be later exchanged by high pressure die casting. The design includes adapters on both plates (see Figure 2a) to connect the three legs out of silicone hoses (see Figure 2b) and lock them firmly with a clip (see Figure 1c) in a notch. The adapters also have undercuts on the inside in order to support the non-adhesive but interlocking properties of the bone cement. After the patient specific pose between the plates is adjusted (compare Section 2.3), hoses are filled with syringes and the cement sets. The silicone hoses are made with a cast, which is realized by rapid prototyping as well. Constant casting results are reached by smooth surfaces, realized with selective laser sintering (SLS) and molds out of polyamide (PA). The final version (shown in Figures 2b and 2c) contains a specialized bellow structure to enable constant diameter without compression (no buckling) and also get a small overall height of the microstereotactic frame. In the operating room, the microstereotactic frame will come in a sterile package (see Fig. 2d). Further details (e.g. material, manufacturer) of the design are listed in Table 1. Failure due to non-completely filled legs or cavities is either detectable by verifying the expected weight of the microstereotactic frame with a scale or fluoroscopy images.

Table 1. Properties of the design of microstereotactic frame.

\begin{tabular}{|c|c|c|c|}
\hline Rapid prototyping material (cast, plates) & PA & \multirow{3}{*}{$\begin{array}{r}\text { Dimensions of plates } \\
\text { top } \\
\text { bottom }\end{array}$} & \multirow{3}{*}{$\begin{array}{l}6 \mathrm{~mm} \text { (height) } \\
70 \mathrm{~mm} \text { (diameter) } \\
90 \mathrm{~mm} \text { (diameter) } \\
\end{array}$} \\
\hline Rapid prototyping technique (cast, plates) & SLS & & \\
\hline Rapid prototyping manufacturer & Materialise & & \\
\hline Manufacturer of bone cement & Heraeus & \multirow[t]{3}{*}{ Dimensions of hoses } & 35 mm (length) \\
\hline Model of bone cement & Palacos LV & & $15 \mathrm{~mm}$ (diameter) \\
\hline Amount of bone cement needed & $40 \mathrm{~g} / 20 \mathrm{ml}$ (1 package $)$ & & $0.4 \mathrm{~mm}$ (thickness) \\
\hline $\begin{array}{l}\text { Initial processing temperature } \\
\text { of bone cement }\end{array}$ & $5^{\circ} \mathrm{C}$ & $\begin{array}{l}\text { Total weight of stereotactic } \\
\text { frame }\end{array}$ & $87 \mathrm{~g}-90 \mathrm{~g}$ \\
\hline Bone cement set time & $16 \mathrm{~min}$ & $\begin{array}{l}\text { Material of silicone } \\
\text { hoses }\end{array}$ & SF - 13 ShA \\
\hline
\end{tabular}



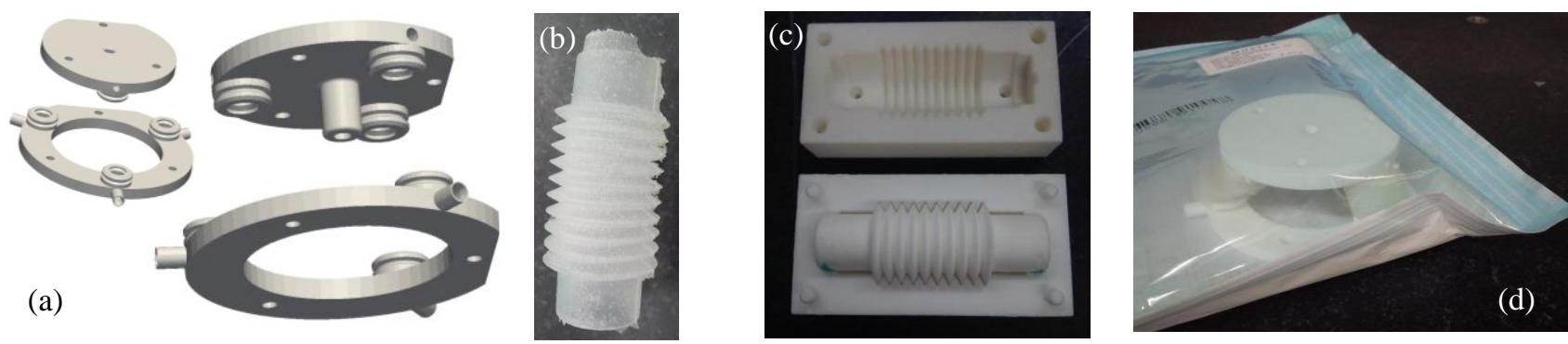

Figure 2. Components, manufacturing and packaging of patient-specific microstereotactic frames. (a) CAD views of the top and bottom plate. (b) Cast silicone hoses, which mount between the two plates. (c) Core and casting molds for the fabrication of silicone hoses. (d) Mockup of sterile packaging for microstereotactic frames.

\subsection{Device to set the pose of the microstereotactic frame inside the operating room}

The device holds one of the two plates of the microstereotactic frame from the top and one from the bottom. A C-shaped stage out of strut profiles was build. The setup with its components and some steps of the workflow are shown in Figure 3. Sterile drapes, which usually are designated for surgical microscopes, are modified by removing central portion of the plastic part for the microscope lens. Although, this is an applicable modification in the operating room for now, we expect a specialized drape in the long run. Two drapes are pulled over the device. A sterile person pulls the sterile drape with the removed section over an adapter with a small rim (which is not sterile). Afterwards, the non-sterile area is covered with another sterile adapter (i.e. a cap), bolt it down and everything is repeated for the second drape. Later on, the sterile adapter might come already bond to the drape. Like with the surgical microscope or an intraoperative C-arm, a second non-sterile person helps to fix the drapes. The design of the mechanical parts includes that the adapters always mount in the same way and no different distances result from the application or production of the drape. A calibration object is placed between the caps instead of the microstereotactic frame, clearances are analyzed and can be corrected.

(a)

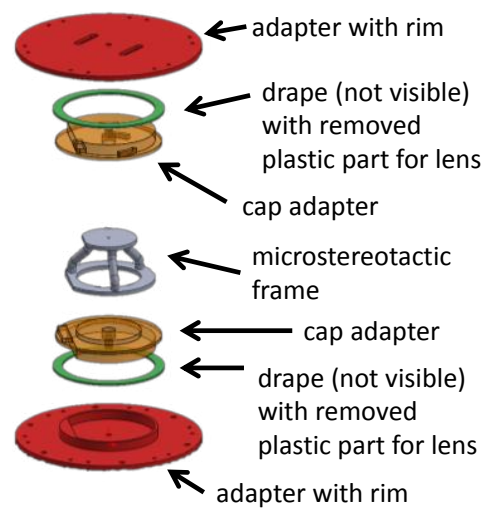

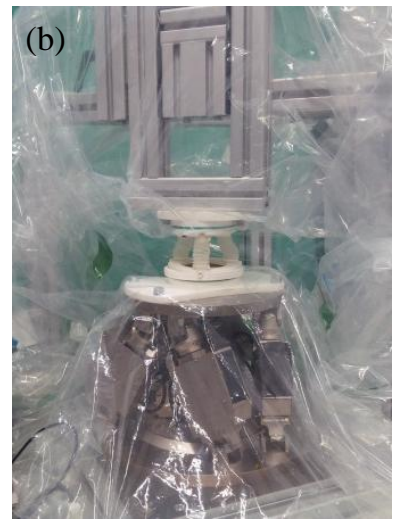

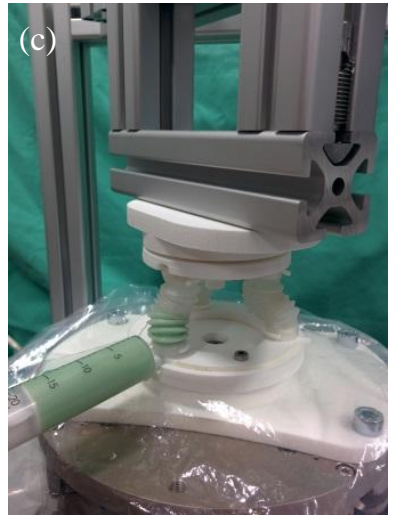

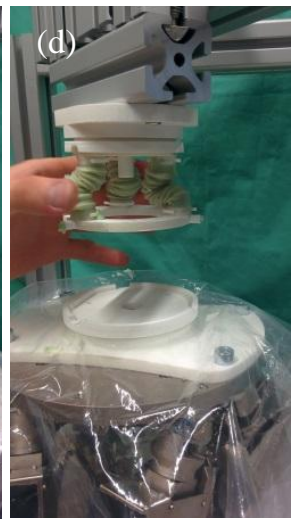

Figure 3. (a) CAD explosion view and (b) photograph of device for setting the pose of the microstereotactic frame. (c) Process of filling the bone cement into the legs. (d) After set time of bone cement microstereotactic frame is ready to use.

Another requirement beneath sterile environment is to set the pose with a high accuracy in at least five DOF. In our first setup, this is realized by a high precision, six DOF actuated system (Hexapod H-824, PI GmbH \& Co. KG) as well as a reproducible mounting of the microstereotactic frame inside the device with a feature fit and little play. As visible in Figure $3 \mathrm{c}$ and $3 \mathrm{~d}$, not all experiments are performed under or simulate sterile conditions at this point of development.

\subsection{Workflow, verification measurements and testing}

19 samples are realized by following the workflow shown in Figure 4. Different poses were created, limited by the parallel kinematics workspace. As already mentioned, the first two steps are not performed every time. At this point of 
research the hoses are not preassembled to the blank frame so that their fixation belongs to the preoperational mounting step.

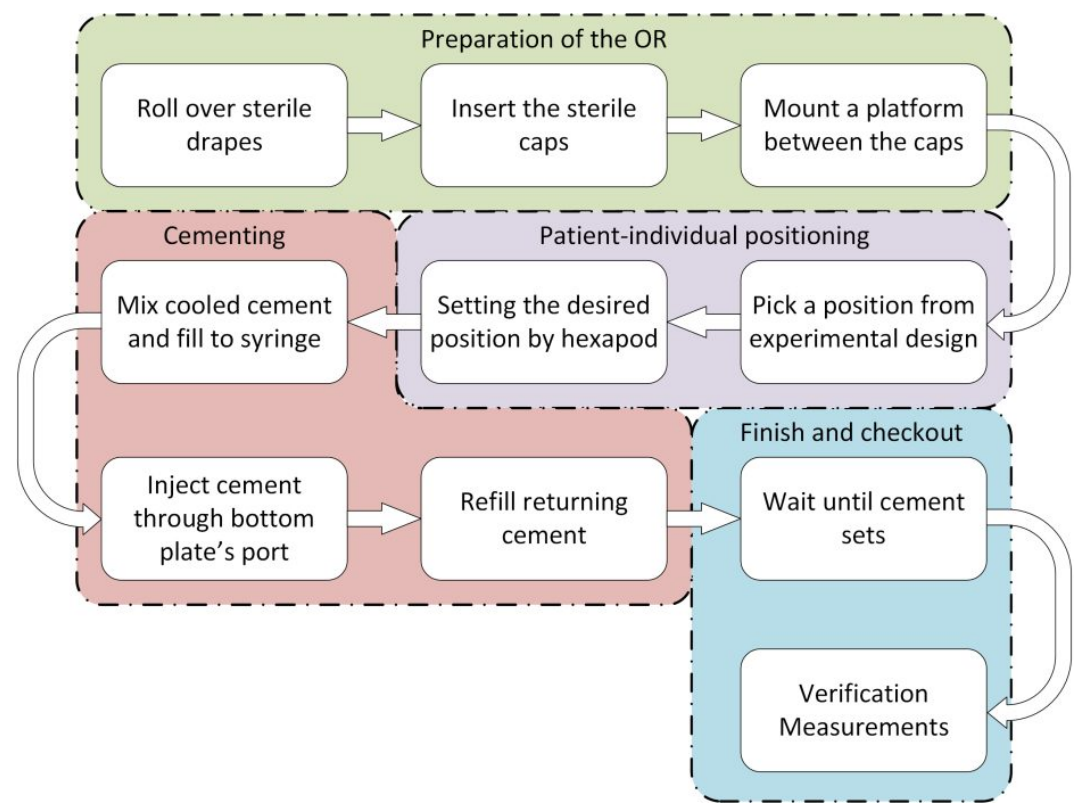

Figure 4. Steps for setting-up, cementing and measuring samples of the microstereotactic design.

For verification purposes, we used a mobile coordinate measuring machine (Gage, Faro Europe GmbH \& Co. KG). After fixation of the frame with bone cement, we measured the relative pose between the adapters (see Figure $3 \mathrm{a}$, after unmounting the microstereotactic frame) and compared it with the resulting pose of the microstereotactic frame. We analyzed several features of the samples like the top and bottom planes, the plates as circles and the trajectory itself as a vector (see Figure 5a). We determine parallel offsets by comparing the nominal and actual intersection points of the trajectory and the lower plate's bottom plane as well as we calculated the deviation that is caused by the relative angular offset of the plates at the end of an expected trajectory length of approx. $85 \mathrm{~mm}$ (resulting from estimated depths of the burr inside the skull and frame height). Few samples that have been fixated in neutral position (home of the parallel kinematics) are used to perform static load measurements with a compression testing machine (Mini Bionix 858, MTS Systems Co.) in three orientations. Figure 5b shows the experimental setup of one lateral direction exemplarily. Based on the recorded fractures during these tests we made fluoroscopy images of intact samples in order to evaluate weak spots that can be caused by low cement levels in the hoses or cavities.

\section{RESULTS}

Figure 6 a shows the measured deviations based on the parallel offset (blue crosses) with worst case added deviations by angular misplacement (red circles). Mean value and standard deviation (green circle, $(0,745 \pm 0,630) \mathrm{mm})$ of the measurements as well as the desired accuracy of $0.5 \mathrm{~mm}$ (dashed black circle) are visualized. We were able to apply the desired angle accurately $(0.079 \pm 0.160)^{\circ}$. Due to varying tolerances (rapid prototyping tolerances) between the caps (aluminum) and blank frames, the experiments result in partial larger errors from not ideally centered initial positions.

Stability experiments gave an estimate of the order of magnitude of the tolerable load. Results are plotted in Figure 6b for exemplarily load cases. None of the tests showed a bigger deformation than $0.20 \mathrm{~mm}$ at a force of $50 \mathrm{~N}$ which is about four to twelve times higher than the expected force resulting from drilling in bone. Samples from early experiments lost their integrity at lower forces than later ones (comparing Specimen 1 and 2 in Figure 6b, top row). All noted fractures invariably occurred in the area of the upper plate's connector and beginning hose. This suggests a weak spot in that zone of the cement.

An exemplary fluoroscopy image (see Figure 7) shows an intact platform from early experiments. Red marked cavities with low density illustrate that no or less cement were present. To prevent these voids, the filling procedure has been 
changed during the development. The frames are now filled by three syringes that stay plugged until the cement reaches a very high viscosity so that no cement returns from the hoses.
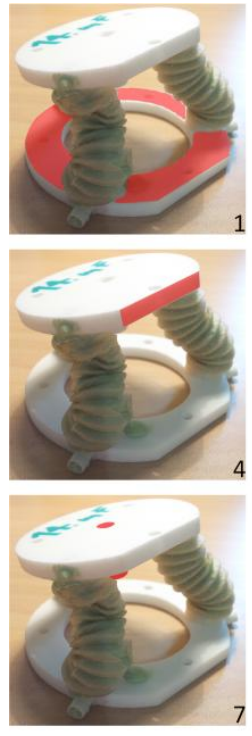
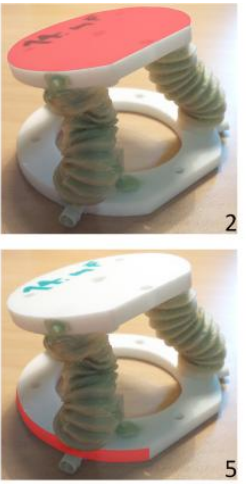

(a)
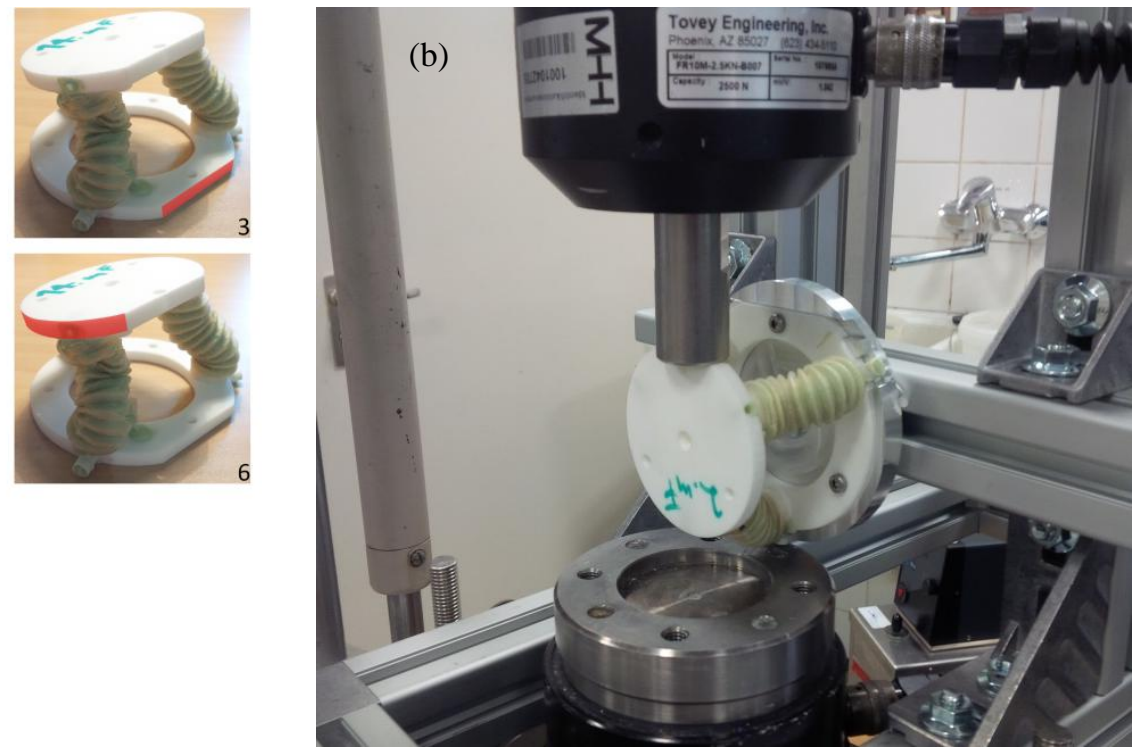

Figure 5. Verification measurements. (a) Measured planes and features of the frame. (b) Exemplary load case of the compression tests.
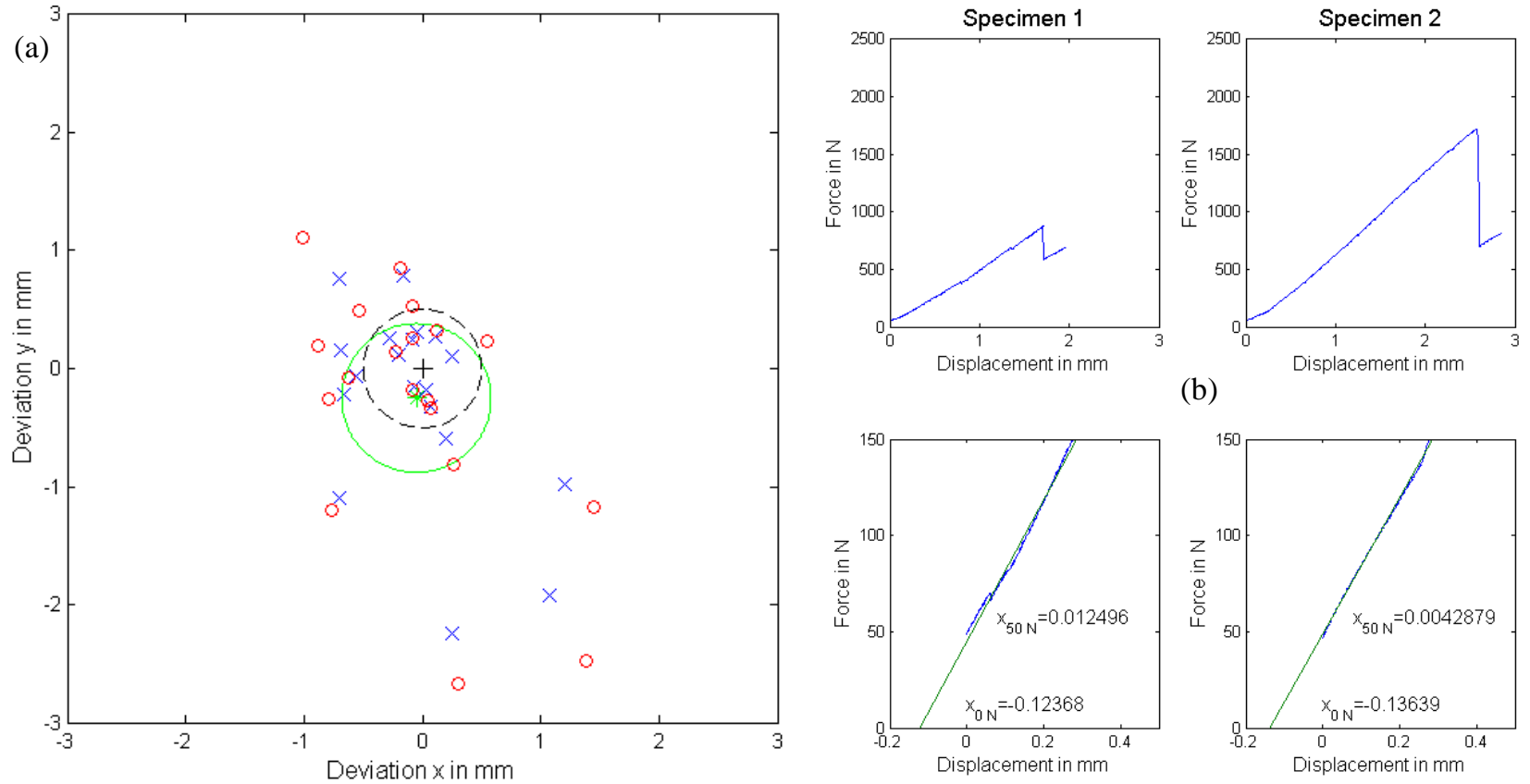

(b)
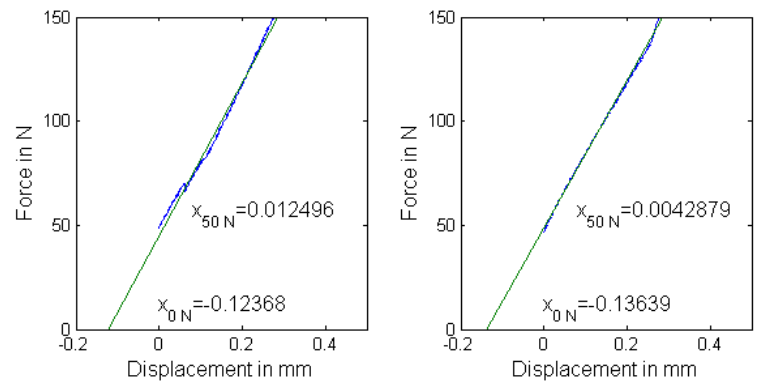

Figure 6. (a) Measured and calculated worst case target point errors. (b) Exemplary compression test results for two specimen. Overall view (top row), detailed plot of low force area (bottom row) 

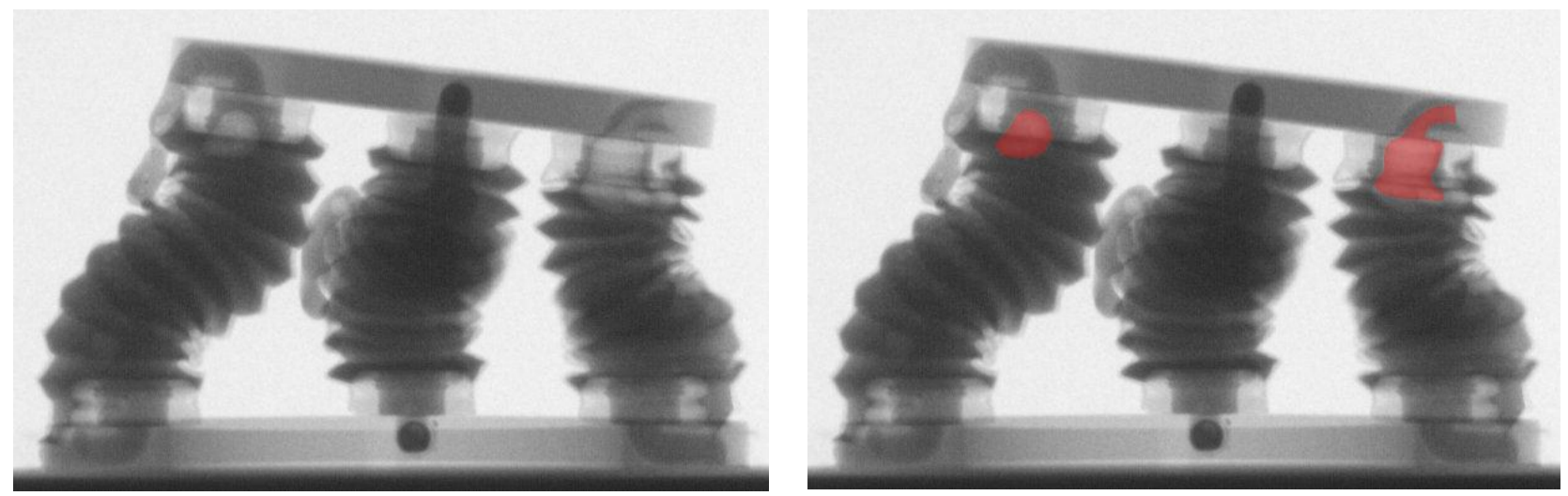

Figure 7. Fluoroscopy control of cement level; original (left image) and segmented volumes (red, right image).

\section{CONCLUSION AND OUTLOOK}

We designed, built and tested intraoperative, adjustable microstereotactic frames based on rapid prototyping parts, silicone hoses and bone cement. Additionally, we developed a setup with a mechatronic high precision parallel kinematics that enables taking the patient-individual pose. It is feasible to cover the unsterile parts of the system with sterile drapes to guarantee direct transfer of the frame to the patient. Up to now, we measured and calculated target point deviations and reproducibility of the stereotactic frames by generating a set of 19 samples.

Our microstereotactic frame competes with others of its kind ${ }^{2,4}$, rapid prototyping solutions $\mathrm{s}^{6,7}$ and further non-disposable platforms ${ }^{8-11}$. Another research group used bone cementfor deep brain stimulation, but performed a fixation of a small block directly to the skull ${ }^{12}$. Our presented design is the first which uses bone cement to build a microstereotactic frame with existing line of sight towards the operational area. It has great potential to be accepted immediately by operating room personnel. Especially the well-known bone cement enables operating room use without further investigation ${ }^{13,14}$. Most cost effective is a design with a small number of thin legs, because of minimum use of bone cement, but further studies are needed to optimize stability and bone cement volume. So far we could figure out that it is applicable to use three legs of a medium diameter $(15 \mathrm{~mm})$ in order to obtain a solid platform that can be filled easily by one person and one package of bone cement.

Beside the cement, other parts (silicone hoses or plastic foil, plates) of the microstereotactic frame are sterilizable before taking them to the operating room. Like with other plastic parts (e.g. drapes), ethylene oxide sterilization is favorable. Other materials can be tested in the future to allow also steam sterilization. We expect a quick transfer of these methods into the operating room because there is neither a time consuming intraoperative sterilization process necessary nor a cutting manufacturing technique used for the presented concept.

In order to reach the expected angles of the trajectory to the skull surface it might be required to change the overall setup for surgical applications with a mechanic or mechatronic system that provides a larger, optimized workspace ${ }^{15}$ than the actual used actuation system. Another option would be to preposition the trajectory by a wedge part or a frame design with an angled guide of the top plate. Furthermore a new test rig that is designed without strut profiles and as few RP parts as possible for less play/tolerance and higher sample accuracies is favorable.

We are certain that the actual production speed of average 25 to 30 minutes can be shortened by experimenting with different cement types and temperatures. Another future idea is to calculate a minimum weight for the frame depending on the desired position (individual leg length and filling volume) and check before mounting it to the head instead of fluoroscopy imaging.

\section{ACKNOWLEDGMENT}

LAK thanks Louis B. Kratchman, J. Michael Fitzpatrick, Ramya Balachandran and Robert F. Labadie from Vanderbilt University, Nashville, TN, for fruitful discussions of further concepts of microstereotactic frames. The authors thank Andre Biewald from Heraeus Medical GmbH for providing samples of bone cement. Many thanks to Ernar Amanov, Carolin Fellmann and Jessica Burgner-Kahrs as well as Mandy Mahzun for their support with the rapid prototyping 
systems and Stefan Düring for his help in the machine shop (all Leibniz Universität Hannover). We are very grateful to Manuel Krämer and the Laboratory for Biomechanics and Biomaterials (LBB) of the Hannover Medical School (MHH) for the help and allocation of their compression testing machine

\section{REFERENCES}

[1] Fitzpatrick J.M., Konrad P.E., Nickele C., Cetinkaya E. and Kao C., "Accuracy of customized miniature stereotactic platforms," Stereotact. Funct. Neurosurg. 83(1), 25-31 (2005).

[2] Labadie R.F., Mitchell J., Balachandran R. and Fitzpatrick J.M., "Customized, rapid-production microstereotactic table for surgical targeting: description of concept and in vitro validation," Int. J. Comput. Assist. Radiol. Surg. 4(3), 273-280 (2009).

[3] Labadie R.F., Balachandran R., Noble J.H., Blachon G.S., Mitchell J.E., Reda F.A., Dawant B.M. and Fitzpatrick J.M., "Minimally invasive image-guided cochlear implantation surgery: First report of clinical implementation," Laryngoscope. 124(8), 1915-1922 (2014).

[4] Kratchman L.B. and Fitzpatrick J.M., "Robotically-adjustable microstereotactic frames for image-guided neurosurgery," Proc. SPIE 8671, 86711U (2013).

[5] Kratchman L.B., Blachon G.S., Withrow T.J., Balachandran R., Labadie R.F., and Webster III R.J., "Design of a bone-attached parallel robot for percutaneous cochlear implantation," IEEE Trans. Biomed. Eng. 58(10), 2904-2910 (2011).

[6] Rajon D.A., Bova F.J. and Friedman W.A., "Rapid fabrication of custom patient biopsy guides," J. Appl. Clin. Med. Phys. 10(4), 2897 (2009).

[7] Cho B., Matsumoto N., Mori M., Komune S. and Hashizume M. "Image-guided placement of the Bonebridge without surgical navigation equipment," Int. J. Comput. Assist. Radiol. Surg. 9(5), 845-855 (2014)

[8] Kobler J.-P., Kotlarski J., Öltjen J., Baron S. and Ortmaier T., "Design and analysis of a headmounted parallel kinematic device for skull surgery," Int. J. Comput. Assist. Radiol. Surg. 7(1), 137-149 (2012).

[9] Thiran J.P., Charrier R., and Haag D., "The stereopod: an accurate guidance system for frameless stereotactic neurosurgery," Int. J. Comput. Assist. Radiol. Surg. 5(S1), 255 (2010).

[10] Joskowicz L., Shamir R., Freiman M., Shoham M., Zehavi E., Umansky F. and Shoshan Y., "Image guided system with miniature robot for precise positioning and targeting in keyhole neurosurgery," Comput. Aided Surg. 11(4), 181-193 (2006).

[11] Nau-Hermes M., Schmitt R., Becker M., El-Hakimi W., Hansen S., Klenzner T. and Schipper J., "Quality Assurance of Multiport Image-Guided Minimally Invasive Surgery at the Lateral Skull Base," BioMed Res. Int., Article ID 904803 (2014).

[12]Eljamel M.S., Tulley M. and Spillane K., "A simple stereotactic method for frameless deep brain stimulation," Stereotact. Funct. Neurosurg. 85(1), 6-10 (2007).

[13] Kuehn K.D., Ege W. and Gopp U., "Acrylic bone cements: composition and properties," Orthop. Clin. North. Am. 36(1), 17-28 (2005).

[14] Kuehn K.D., Ege W. and Gopp U., "Acrylic bone cements: mechanical and physical properties," Orthop. Clin. North. Am. 36(1), 29-39 (2005).

[15] Kobler J.-P., Kotlarski J., Lexow G.J., Majdani O., and Ortmaier T., "Design Optimization of a Bone-Attached, Redundant and Reconfigurable Parallel Kinematic Device for Skull Surgery," Proc. IEEE Int. Conf. Robot. Autom., 2364-2371 (2014). 